\title{
What can gait tell us about dementia? Review of epidemiological and
}

\section{$\underline{\text { neuropsychological evidence }}$}

\section{$\underline{\text { Abstract }}$}

Background: Cognitive impairment and gait disorders in people over the age of 65 represent major public health issues because of their high frequency, their link to poor outcomes and high costs. Research has demonstrated that these two geriatric syndromes are closely related. Methods and Results: We aim to review the evidence supporting the relationship between gait and cognitive impairment, particularly focusing on epidemiological and neuropsychological studies in patients with Mild cognitive impairment, Alzheimer's disease and Vascular dementia. The review demonstrates that gait and cognition are closely related, but our knowledge of their interrelationship is limited. Emerging evidence shows that gait analysis has the potential to contribute to diagnosis and prognosis of cognitive impairment.

Conclusions: An integrated approach for evaluating these major geriatric syndromes, based on their close relationship, will not only increase our understanding of cognitive-motor interactions, but most importantly may be used to aid early diagnosis, prognosis and the development of new interventions.

Key words: gait, cognition, cognitive impairment, Alzheimer's disease, Vascular dementia

\section{1 words}




\section{Introduction}

Cognitive impairment and gait disorders in people over the age of 65 represent major public health issues because of their high frequency, their link to poor outcomes and high costs [1].

Gait changes with ageing [2]. A gait disorder is present when the patient walks even more slowly than expected for age, or when there are qualitative abnormalities of locomotion, such as disturbances of the initiation of gait or balance [2]. Gait dysfunction and gait abnormality are terms describing deviation from normal walking, but not to the degree of a recognised gait disorder. Motor dysfunction is a broader term, including not only impairment in gait, but other motor skills as well.

We describe the evidence supporting the relationship between gait and cognition, possible shared pathomechanisms and the gait changes associated with Mild Cognitive Impairment (MCI) and Dementia. We review the evidence suggesting that gait performance is a predictor of adverse outcomes, particularly cognitive decline and conclude by outlining the role of interventions and future directions in this growing area of research.

\section{Methods}

We searched PubMed from 1970 to October 2016 for the following terms and keywords: 'gait', 'walking', 'gait analysis', 'gait disorder', 'gait variability', 'gait measures', 'mobility', 'ageing', 'cognition', 'cognitive impairment', 'dementia' and 'older adults'. 1203 titles and abstracts were screened and we then reviewed 171 full text papers. We included epidemiological and neuropsychological studies that investigated the relationship between gait and cognition in healthy older adults, as well as in people with MCI, Alzheimer's disease (AD) and Vascular dementia (VD), particularly when caused by small vessel disease (SVD). 
Studies in disorders associated with well-characterised motor symptoms were excluded, as these are beyond the scope of the review. We identified additional studies by hand-searching reference lists (Fig 1).

\section{Results}

\section{$\underline{\text { Relationship between gait and cognition }}$}

Human gait is considered a complex, non-linear process during which the locomotor system incorporates input from the cerebellum, the motor cortex, and the basal ganglia, as well as feedback from visual, vestibular and proprioceptive sensors [3]. Converging evidence suggest that gait also relies on a higher order cognitive control [4]. Because intact walking requires coordinated function of multiple regulatory circuits, deviation from the normal walking pattern is a sensitive marker of failure in any of the circuits.

The cortical involvement in gait control is highlighted by studies using the dual task paradigm, which is based on the hypothesis that two simultaneously-performed tasks interfere if relying on identical functional subsystems. The dual task effect, which represents the change in gait pattern between dual and single task performance or change in absolute performance under dual task conditions, is observed in healthy adults, but it increases with age and in subjects with cognitive impairment. The magnitude of the effect is directly related to the degree of cognitive impairment [5-8]. It has been postulated that impaired cognition results in reduced attentional resource allocation, which can compromises gait stability [9].

\section{Possible shared pathomechanisms underlying the relationship between gait and} cognition 


\section{- Neurodegeneration}

Neurodegeneration in multiple systems leading to coexisting motor and cognitive symptoms is a mechanism common to several neurological disorders [10]. Little is known about the underlying pathophysiology of the motor symptoms in early AD. Later, when the degenerative process spreads to the frontal areas of the brain, gait apraxia is a common finding [11]. Contrary to the original view of AD as involving mainly cortical degeneration, systematic pathological studies have found considerable involvement of brainstem nuclei, which by means of their widespread afferent projections can modulate disparate brain regions to generate integrated motor and cognitive responses [12]. Degeneration in multiple systems is thus likely to underlie the motor symptoms in AD.

\section{- Inflammation}

Another pathological process, common to ageing and associated with decline in gait speed and memory impairment is low-grade systemic inflammation characterised by increased proinflammatory markers [13]. Inflammation is present in most neurological diseases manifesting with impairment of mobility and cognition: it has been implicated in development and progression of AD. Elevated inflammatory markers and activated microglia are found in both disorders $[13,14]$, as it is in vascular cognitive impairment [15]. Neuroinflammation, manifesting as microglial activation, may lead to increased oxidative stress, pathologic synaptic pruning and impaired neuroplasticity in key brain regions sub-serving cognition and motor function.

\section{- Vascular}


Of particular importance is vascular burden $[13,16]$. While vascular damage is the main causal factor in VD, it also plays a key role in normal ageing and in the development and progression of cognitive, affective and motor symptoms associated with other disorders [16]. Vascular risk factors, including hypertension, diabetes, hypercholesterolemia and atrial fibrillation, among others, confer increased risk for developing of not only VD but also $\mathrm{AD}$, with mixed pathologies present in a significant proportion of cases [17]. Vascular risk factors are associated with white matter changes, which are associated with gait slowing (defined as gait velocity $<0.5 \mathrm{~m} / \mathrm{s}$ ) in cognitively healthy older adults [18].White matter changes also represent a marker for current and future cognitive and gait impairment $[19,20]$.

A possible unifying underlying pathological mechanism could be the age-related diffuse micro-damage to small vessels, which compromises the integrity of the frontalsubcortical circuits involved in the cortical control of motor behaviour and in complex cognitive functions [16]. While there is an empirical association between vascular risk factors, cognitive and gait impairment, it remains difficult to identify the biological mechanisms linking these conditions.

\section{$\underline{\text { Relationship between neuropsychological performance and gait }}$}

\section{- Cognitively healthy older people}

In healthy older adults, there is evidence of a small positive association between gait and global cognition (12 studies, $\mathrm{d}=0.12,95 \% \mathrm{CI}=0.09$ to $0.15, p<.001)$ [21]. In this metaanalysis, most studies used speed of gait as the outcome measure, while the most common 
test of global cognition was the MMSE (Mini Mental State Examination, with a score below 24 denoting cognitive impairment).

Neuropsychological studies have consistently demonstrated a positive association between executive functions and gait measures in cognitively healthy older people [22-28], although there are some studies that show no association $[29,30]$. For example, worse executive function has been associated with decreased gait velocity [23, 26], slower pace [27] and reduced cadence [24]. A meta-analysis of 18 studies revealed a moderate pooled association between gait and measures of executive function $(\mathrm{d}=0.2,95 \% \mathrm{CI}=0.15$ to $0.26, p<0.001)$ [21].

Executive function is also correlated with gait variability $[31,32]$. For instance, the degree of executive dysfunction was correlated with the degree of stride time variability (STV)[22]. STV is a sensitive marker of gait stability [33]. It has been suggested that it reflects one of the final most highly integrated CNS pathways of gait [33]. In healthy adults, STV is less than 3 $\%$ [34], while higher STV has been found in frail older adults [35]. Lower STV is associated with efficient and safe gait patterns, and it reflects automatic processes that require minimal conscious cortical input [33]. If the automatic processes are disrupted, intact executive function is required to compensate.

Other cognitive domains, beyond executive function, are involved in gait control. Immediate memory, which is often considered a construct of attention and executive function, has also been linked to gait dysfunction [23, 25, 28, 36, 37] and falls [38] in older adults. More specifically, in healthy elderly, worse memory was associated with reduced cadence [25], and impaired gait velocity in single [23] and dual task conditions [25, 37]. A significant number 
of studies, however, did not show association [22, 26, 27, 30, 39], suggesting that further research is required. A meta-analysis including 10 studies reported small association between greater gait speed and better performance on memory tests $(\mathrm{d}=0.14,95 \% \mathrm{CI}=0.1$ to $0.19 ; p<$ $0.001)[21]$.

There is less convincing evidence for an association between performance in the visuospatial domain and gait in older adults. Visuospatial deficits were associated with reduced gait velocity [30,36], as well as with increased double support phase variability [26], which in turn is associated with an increased risk of falls [40].

Some studies have also found an association between language and gait velocity $[23,36]$ or pace [24]. Decreased processing speed has been found to correlate with poorer performance in many gait measures, including gait velocity [23, 26, 30], rhythm [27], step time, step length, and double support phase variability [26]. A meta-analysis of 9 studies reported a small association between reduced gait performance and lower processing speed $(d=0.15$, $95 \% \mathrm{CI}=0.1$ to $0.2 ; p<0.001)$ [21]. Overall, the research into the relationship between gait and specific cognitive domains other than executive function is limited and predominantly restricted to speed-related outcomes. This is particularly true in clinical samples.

\section{- Cognitively impaired older people}

MCI is a term describing impairment in one or more cognitive domains that is greater than normal age- related changes, but not of the magnitude to warrant a diagnosis of dementia [41]. In dementia, the cognitive impairment is severe enough to interfere with normal activities of daily living. Executive dysfunction has been associated with reduced gait speed and increased variability in studies in patients with MCI [42, 43] and AD [31, 43], although 
Sheridan et al. 2003 could not confirm this in 28 patients with probable AD [44].

Interestingly, impaired executive function significantly modified STV under single and dual task conditions. In subjects with dementia and dysexecutive syndrome, when they were compared to healthy subjects and subjects with dementia, but without executive dysfunction [45]. Decline in executive function, but not other cognitive domains, was also longitudinally associated with decline in gait speed $(\beta=-3.55,95 \% \mathrm{CI}=-5.49,-1.61 ; p<.001)$, both in the presence and absence of baseline cognitive impairment [46].

In people with amnestic and non-amnestic MCI, lower episodic memory performance on the Rey Auditory Verbal Learning Test (RAVLT) was linked to an increased dual task cost [47]; dual task cost represents the deterioration in performance under dual-task conditions and is adjusted for individual baseline gait characteristics. In this study, the authors controlled for the executive dysfunction present in the amnestic MCI group, but not for the global degree of cognitive impairment, making the interpretation of the results difficult.

\section{Gait characteristics associated with MCI}

There are limited studies evaluating gait in MCI, with most [6, 7, 47-54] but not all of them [55-58] finding evidence of gait dysfunction under normal walking conditions.

Pettersson et al. (2005) did not find differences in motor function when comparing people with MCI to controls [57]. However, the study used a heterogeneous MCI group, including people with single-domain and multi-domain MCI, while there is evidence that the gait patterns differ between MCI subtypes [47, 48]. Further, performance-based tests were used to 
assess motor function and a ceiling effect was observed, suggesting that the study lacked sensitivity to detect subtle changes.

When quantitative gait measures are used the results of individual studies are not always consistent, but the pooled effect sizes support the presence of gait impairment in MCI [59]. Bahureksa et al., 2016 included 11 studies in a meta-analysis and found that under single task conditions velocity $(d=-0.74, p<0.01)$, stride length $(d=-0.65, p<0.01)$, and stride time (mean: $\mathrm{d}=0.56, \mathrm{p}=0.02$; coefficient of variation: $\mathrm{d}=0.50, \mathrm{p}<0.01$ ) discriminated best between MCI and healthy controls [59]. Further, Beauchet et al. (2014) demonstrated that higher STV was related to both MCI (effect size $=0.48$ [95\% CI: $0.30 ; 0.65])$ and dementia (effect size 1.06 [95\% CI: 0.40; 1.72]) in a meta-analysis of eight studies [34]. Higher STV, which as described above represents a fine marker of gait control, has also been associated with episodic memory impairment and executive dysfunction in a community study of 934 people without dementia; the authors concluded that higher STV appears as the motor phenotype of cognitive decline [34].

Importantly, Bahureksa et al (2016) found that DT assessment increased the discriminative power of gait variables and several studies have found gait changes in MCI under dual task conditions only $[56,58,59]$. Gait variability may be especially sensitive to dual task, as a significant dual task x cognitive status interaction was found for gait variability, but not for gait velocity, when older adults with MCI were compared with controls [7].

Quantitative gait analysis has also shown gait dysfunction in patients with both amnestic MCI and non-amnestic MCI [48]. Abnormal gait was almost twice as common in amnestic MCI as in normal controls [31.5\% vs. $16.3 \%$ ]. Factor analysis of gait measures in patients with 
amnestic MCI, non-amnestic MCI and controls, revealed three independent factors representing pace (a measure of velocity and stride length), rhythm (depends on swing and cadence) and variability. In non-amnestic MCI, the pace factor was more affected, while in amnestic MCI the rhythm factor and variability measures were worse. Interestingly, the pace factor was associated with longitudinal decline in executive functions, whereas rhythm predicted a decline of episodic memory, thus providing evidence for specific links between gait measures and cognitive domains [60].

\section{Gait characteristics associated with AD}

Gait disorders are more prevalent in people with dementia compared with people without dementia, and there is a direct relationship between the severity of cognitive impairment and increased gait abnormalities [61-64].

Mild AD is not thought to be associated with gait disorders. The NINCDS-ADRDA criteria postulate that gait disorders early in the course of the disease make the diagnosis of probable AD unlikely [65]. In a validation study of these criteria, Ala and Frey (1995) looked for documented gait impairment at first presentation of autopsy-proven AD [66].Gait abnormalities were not reported by any of the patients with mild AD, while they were experienced by $16 \%$ and $32 \%$ of patients with moderate or severe disease, respectively. Using clinical assessment (i.e. the Tinetti gait protocol) to investigate the presence of gait disorder in $\mathrm{AD}$ and non-AD dementia, including $\mathrm{VD}$, no gait disorders were found in early $\mathrm{AD}$, although they were observed in moderate and severe $\mathrm{AD}$ [62]. The methodology in both studies, for example self-report in Ala and Frey (1995) and focus on recognised gait disorders, rather than specific gait abnormalities in Allan et al. (2005), may have resulted in 
limited sensitivity to early gait changes. When the focus was on identifying gait dysfunction, the Tinetti test was abnormal in $31.5 \%$ of patients with mild-to moderate AD, implying that there is a degree of gait impairment earlier in the disease course [67]. Further, Pettersson et al. (2002) found that, when compared to controls, patients with mild AD had lower scores on the Berg Balance Scale, they took longer to complete the timed Up-and-Go test, and took more steps outside the figure, when asked to walk in a figure of eight [68]. Using the Tinetti scale, gait dysfunction was found to occur 4.19 years (median time) after AD onset [69].

The presence of gait disorders in later stages of dementia is well established $[61,70,71]$. In contrast to the 'cautious gait' pattern observed in early AD, patients with severe dementia suffered from various gait disorders, including frontal gait disorder, characterised by shuffling and hesitation in starting and turning $[72,73]$.

When more sensitive tools for spatiotemporal gait assessment are used, consistent differences are found. Early in the disease course, people with AD demonstrate accelerated decline in gait measures typically declining with age. They show cautious gait, which is defined as decreased gait velocity, impaired static and dynamic balance and shorter and more variable stride length $[8,52,61,70,72-75]$.

Similar to people with MCI, dual-tasking increases the sensitivity of gait assessment, with some studies finding gait changes such as decreased velocity, increased stride time and STV in dementia under dual task conditions only [58, 76]. Gillain et al. (2009) found that during the transition from single to dual task conditions, patients with AD demonstrated a significant decrease in gait speed and other gait parameters indicating deterioration in gait performance [77]. These findings are supported by other studies in $\mathrm{AD}[44,78,79]$, as well as in a 
population of subjects presenting with $\mathrm{AD}, \mathrm{VD}$ or mixed dementia and frontal impairment [80].

Van Iersel et al. (2004) conducted a systematic review of 7 studies that compared people with AD to healthy older adults, using quantitative gait analysis or mobility assessment scales [61].They found consistent changes in gait measures, such as reduced speed and decreased step length despite the heterogeneity of methods used. Stride-to-stride variability was only measured in two studies, but it seemed increased compared with normal controls. Despite the reduced gait speed, and considering their overall level of physical disability, patients with AD walk too fast [81].The relatively fast gait speed in patients with dementia may reflect frontal lobe disinhibition or lack of insight [1] and probably contributes to the increased risk of falls in this population.

\section{Gait characteristics associated with small vessel disease (SVD)}

SVD, including combinations of lacunae and white matter lesions, even if not severe enough to cause dementia, is related to cognitive impairment and gait disturbance [82]. Two recent systematic reviews found that white matter hyperintensities (WMH) burden is associated with gait disorders $[19,83]$. More specifically, white matter lesions in the frontal lobe, the centrum semiovale, the internal capsule and the corpus callosum were related to gait dysfunction. The gait parameters frequently linked to WMH included gait velocity, stride length and step width [19]. Longitudinal studies show that both baseline WMH and progression of WMH predict increased risk of falls [84-86]. 
Despite these consistent results, studies on the relationship between WMH and gait have methodological limitations. For instance, De Laat et al. (2010) found that white matter lesions and lacunar infarcts were both independently associated with most gait parameters, with stride length being the most sensitive [87]. WMLs in the basal ganglia, internal capsule and limbic areas, as well as lacunar infarcts in the frontal lobe and thalamus were related to a lower velocity [87]. Study participants may have had MCI as they were screened with the MMSE only, which is not a sensitive test [88]. Similarly, decline in gait and balance performance were correlated with the severity of white matter changes in nondisabled community elderly [89]. However, the sample was not population based and was heterogeneous, including people referred to Neurology because of cognitive decline, minor strokes, gait disturbances or depression.

\section{Gait characteristics associated with Vascular Cognitive Impairment (VCI) and}

\section{$\underline{\text { Vascular Dementia (VD) }}$}

Gait disturbance is almost invariably present early in the course of VD and is included in the NINDS - AIREN criteria as a symptom consistent with the diagnosis of probable VD [90].

Using quantitative gait analysis, Bazner et al., (2000) established that, patients with subcortical VCI had reduced cadence, increased variability of single and double support times, as well as reduced single support phase [91].

Confirming that more severe disease is associated with more prominent gait changes, a longitudinal study of 1155 patients with subcortical VD found a significant correlation between the Tinetti scale and the Clinical Dementia Rating Scale, with marked decrease in 
gait measures during follow-up [92]. In patients with VD, there is consistent evidence for slow, short-stepping, and wide-based gait, as well as disturbance in initiation of gait and difficulty turning $[72,73]$.

When compared to subjects with AD, subjects with VD walked more slowly and had a reduced step length [62]. In this study, the Tinetti scale was used, and 79 percent of patients with VD exhibited gait and balance disorders, compared with 25 percent of patients with AD. The rate of decline of mobility also differs, depending on the dementia subtype and rate of progression. In a longitudinal study of 766 older adults, all types of dementia showed decline of mobility, but those progressing to non-AD dementia, especially VD, declined faster than those who remained cognitively healthy (slope $=-2.70, \mathrm{P}<0.001$ ) or progressed to $\mathrm{AD}$ (slope $=-$ $2.18, \mathrm{P}<0.001)$. A steeper physical decline was observed in patients with the fastest progression of dementia [93].

\section{$\underline{\text { Association between cognitive impairment, gait impairment and falls }}$}

Cognitive and gait impairment are independent risk factors for falls [94]. The incidence of falls is higher in MCI [95-97], as well as in dementia generally; in subjects with AD and VD it is almost two times higher than in subjects without cognitive impairment [98].

The mechanisms underlying the observed associations are not entirely understood. Similarly to the general older population, falls in dementia are likely to be multi-factorial in origin [98].

There is strong evidence that even subtle cognitive deficits play a significant role. Interference under dual task conditions is a marker of future falls $[7,94,99,100]$, for both 
cognitively intact and impaired $[101,102]$. Other factors may be important: dual task cost was found to predict falls in the short-term in robust older people only (i.e. not frail or cognitively impaired)[103], while other studies found that dual task tests were better in predicting falls in frailer older adults $[99,104]$.

Regarding specific cognitive domains, in cognitively healthy elderly, executive dysfunction has been associated with an increased risk of falling in a meta-analysis of 27 studies [Odds Ratio: 1.44, 95\%CI (1.20-1.73)] [105]. Impairment in attention [4], processing speed [106] and visuospatial performance $[26,107]$ have been linked with higher fall rates in this population. In MCI and dementia, executive and visuospatial dysfunction have been associated with higher fall rates [96, 107]. Amongst gait parameters, reduced velocity and mean stride length were the best predictors of falls in nursing home residents with dementia [108].

\section{Gait dysfunction as a predictor of cognitive decline}

There is increasing evidence that gait dysfunction can independently predict cognitive decline $[60,109-113]$, as well as other adverse outcomes such as disability [114], cardiovascular disease [115] and survival [116].

Several excellent recent reviews have summarised the consistent evidence that poor gait performance predicts cognitive decline [117-119]. Kikkert et al. (2016) reviewed longitudinal studies and concluded that gait slowing $(0.68-1.1 \mathrm{~m} / \mathrm{sec})$ preceded cognitive decline and the development of dementia syndromes [119]. A meta-analysis of twelve studies found that poor gait performance, defined by underperformance on standardised tests of walking, predicted 
dementia with pooled Hazard Ratio (HR) of 1.53 for any dementia $(p<0.001), 1.03$ for AD $(\mathrm{p}=0.004)$ and 1.79 for $\mathrm{VD}(\mathrm{p}<0.001)[117]$. Morris et al. (2016) considered specific gait measures and reported that pace predicted decline in global cognition in seven out of nine studies; only two studies assessed rhythm and their results are contradictory [118]. Gait may change up to 12 years before the clinical presentation of cognitive changes in elderly who later develop MCI [120].

Recently, the concept of Motoric Cognitive Risk syndrome (MCR) has been proposed. MCR is defined as the presence of self-reported cognitive complaints (but no dementia) and slower gait (one standard deviation below age- and sex-specific gait speed means). Older participants who met the criteria for MCR had a higher risk of developing dementia and VD with an adjusted HR of 3.27 and 12.81, respectively [121]. In a further study, including 26,802 adults from 17 countries, the pooled MCR prevalence was $9.7 \%$ [122]. In the pooled sample, MCR was associated with a 2-fold increased risk of developing incident cognitive impairment (aHR 2.0, 95\% CI 1.7-2.4) and dementia (aHR 1.9, 95\% CI 1.5-2.3) in 4,812 participants with baseline MMSE scores $>25$, even after accounting for vascular disease and baseline cognitive status. Importantly, MCR was a stronger predictor of cognitive outcomes than its individual components of cognitive complaints or slow gait. In the validation study, MCR did not predict the risk of $A D$ in the whole sample, but did predict $A D$ in more than 2,000 participants from two cohorts in this study [122].

\section{Interventions based on the interrelationship between gait and cognition to prevent} $\underline{\text { mobility decline or cognitive impairment }}$ 
It is well known that physical activity protects against decline of mobility, and that mentally stimulating activities are beneficial to cognition. However, based on the close relationship, effective interventions may include enhancing cognition to prevent mobility decline or physical activity programmes to delay the onset or progression of cognitive impairment [4].

\section{- Physical activity}

A large meta-analysis has demonstrated that physical activity is inversely associated with dementia risk [123]. Exercise has been shown to improve cognitive functions such as memory, processing speed, attention, and executive function in cognitively healthy adults, as well as in people with MCI, although a significant proportion of studies included in two reviews did not find any effect $[124,125]$, with the results in people with dementia being even less consistent [125]. A recent meta-analysis however showed that aerobic exercise led to an improvement in global cognition and memory in patients with MCI [126]. Interpreting the results of these meta-analyses is difficult because of the significant heterogeneity between the included studies. There was a great variability in the types of exercise used (including aerobic exercise, strength or balance exercise), the program duration ranged from 6 weeks to 52 weeks, the intensity of exercise was operationalized in different ways (for example a heart reserve of $60-80 \%$ or $30-40 \%$ of maximum heart rate), the duration of the sessions ranged from 20-65 min, while the frequency ranged from once per week to five times per week [124, 126]. In addition there was a wide variety of cognitive measurement tools used, and diverse interventions in the control group.

Larger and good quality clinical trials are needed to confirm these findings and to understand whether the beneficial effects on cognition will translate to improved activities of daily living $[4,13,94]$. 


\section{- Cognitive training}

A few studies have evaluated the effectiveness of cognitive interventions, mainly based on dual task training, in improving gait and balance in elderly or cognitively impaired patients. Although the results are generally promising, suggesting that cognitive training can improve gait and balance and lower dual task costs, they are limited by significant heterogeneity between studies, relatively small sample sizes and the fact that they did not investigate the risk of falls per se [94]. Also, most interventions included motor training in addition to the cognitive training, making the separation of their relative contribution to the observed improvement in gait difficult.

The neurobiological basis of the observed motor or cognitive improvement following cognitive training or exercise programs is largely unknown. The interventions may have a direct effect on the brain, by improving the quality of white matter or inducing neurogenesis and brain plasticity, respectively. Neuroimaging provides evidence of experience- dependent structural brain changes in the human brain regardless of age [127]. Indirect effects, related to general improvement of cardiovascular risk status or non-specific effects on motivation and emotion as a result of social engagement cannot be excluded.

\section{- Effect of anti-dementia drugs on gait}

Cholinesterase inhibitors and Memantine are approved for symptomatic treatment of AD. Correcting the cholinergic loss with cholinesterase inhibitors or enhancing the glutamatergic and dopaminergic neurotransmission with Memantine may improve gait performance directly or, alternatively, through improving cognition. A few small studies have evaluated their effect on motor function. The results so far are inconclusive. 
A pilot study found that after treatment with Donepezil gait improved in six patients with AD [128]. Further studies in AD reported improvement in gait velocity [129] and dual task performance [130] but not in STV [129-131] after treatment with cholinesterase inhibitors. Memantine-related decrease in STV was reported by two studies in mild-to-moderate AD, with a total of 126 patients $[131,132]$. However, a meta-analysis found that the decrease in STV with anti-dementia drugs was not significant [133]. The small sample sizes and methodological limitations, including non-randomised study design make the interpretation of the results difficult.

\section{- Non-invasive brain stimulation techniques}

Non-invasive brain stimulation techniques, such as rTMS (repetitive transcranial magnetic stimulation), also have a potential therapeutic utility in the treatment of cognitive and motor deficits $[134,135]$. rTMS is known to modulate cortical excitability and to induce longlasting neuroplastic changes that underlie cognitive and motor learning [134]. A therapeutic effect can be obtained by increasing the excitability of damaged areas or by decreasing the excitability of unaffected areas [136]. Research to date has focused mainly on the effects of rTMS on motor symptoms in PD or after stroke, or its effects on cognitive measures in patients with AD [134-136]. A meta-analysis of 7 studies found that rTMS stimulation has significant therapeutic effect on cognition in patients with mild to moderate AD [134]. Considering that gait and cognitive impairment most likely share causes of pathology, it may be speculated that rTMS represents a potential therapeutic tool to improve cognition by treating motor symptoms or vice versa.

\section{Conclusions}

This review demonstrates that gait and global measures of cognition that lack sensitivity are closely related, but our knowledge of their interrelationship is limited. Emerging evidence 
shows that gait analysis has the potential to contribute to diagnosis and prognosis of cognitive impairment. The progress of our understanding is hindered by the lack of sensitive quantitative gait assessment in clinical practice. To complicate matters further, the diagnosis of cognitive impairment and dementia subtype, even when established criteria are used, is not unequivocal. Many studies have used not very sensitive global measures of cognition, making it likely that participants with milder cognitive deficits were included in the control group. Furthermore, neuroimaging was not always used to support the diagnosis, allowing subclinical cerebrovascular disease to compound the results. Increasing the availability of user friendly gait assessment in clinical practice and improving the accuracy of dementia diagnosis, for example by using biomarkers, will strengthen this area of research.

Shared pathological mechanisms and the relative contribution of cognitive impairment and gait dysfunction to decline with ageing are critical areas. They directly address the degree to which aging and neurodegeneration associated with cognitive or motor impairment are overlapping phenomena and can be ameliorated by common means. Treating reversible vascular risk factors early, for example, has the potential to prevent cognitive and mobility decline. An integrated approach for evaluating these major geriatric syndromes, based on their close relationship, will thus not only increase our understanding of cognitive-motor interactions, but most importantly can be used for the development of new interventions.

\section{$\underline{\text { Authors' Roles }}$}

VV contributed to concept; produced first draft of the paper; and contributed to revisions and final version of the paper. KPE contributed to concept; revisions and final version of the paper. 


\section{Funding sources for study}

This research did not receive any specific grant from funding agencies in the public, commercial, or not-for-profit sectors.

\section{Conflict of interest statement}

VV- none; KPE received grant and other support from the Medical Research Council (United Kingdom), Gordon Edward Small Charitable Trust, Norman Collisson Foundation, HDH

Wills 1965 Charitable Trust, and National Institute for Health Research (England).

\section{Acknowledgement}

Not applicable

\section{$\underline{\text { References }}$}

1. A.H. Snijders, C.C. Verstappen, M. Munneke, and B.R. Bloem, Assessing the interplay between cognition and gait in the clinical setting. J Neural Transm (Vienna), 114 (10) (2007) 1315-21.

2. K. Jahn, A. Zwergal, and R. Schniepp, Gait disturbances in old age: Classification, diagnosis, and treatment from a neurological perspective. Dtsch Arztebl Int, 107 (17) (2010) 306-15; quiz 316.

3. J.M. Hausdorff, Gait dynamics, fractals and falls: Finding meaning in the stride-to-stride fluctuations of human walking. Hum Mov Sci, 26 (4) (2007) 555-89.

4. M. Amboni, P. Barone, and J.M. Hausdorff, Cognitive contributions to gait and falls: Evidence and implications. Mov Disord, 28 (11) (2013) 1520-33.

5. J. Verghese, G. Kuslansky, R. Holtzer, M. Katz, X. Xue, H. Buschke, et al., Walking while talking: Effect of task prioritization in the elderly. Arch Phys Med Rehabil, 88 (1) (2007) 50-3.

6. M. Montero-Odasso, H. Bergman, N.A. Phillips, C.H. Wong, N. Sourial, and H. Chertkow, Dual-tasking and gait in people with mild cognitive impairment. The effect of working memory. BMC Geriatr, 9 (2009) 41.

7. M. Montero-Odasso, S.W. Muir, and M. Speechley, Dual-task complexity affects gait in people with mild cognitive impairment: The interplay between gait variability, dual tasking, and risk of falls. Arch Phys Med Rehabil, 93 (2) (2012) 293-9.

8. Y. Cedervall, K. Halvorsen, and A.C. Aberg, A longitudinal study of gait function and characteristics of gait disturbance in individuals with alzheimer's disease. Gait Posture, 39 (4) (2014) 1022-7. 
9. M. Woollacott and A. Shumway-Cook, Attention and the control of posture and gait: A review of an emerging area of research. Gait Posture, 16 (1) (2002) 1-14.

10. N.I. Bohnen, K.A. Frey, S. Studenski, V. Kotagal, R.A. Koeppe, P.J. Scott, et al., Gait speed in parkinson disease correlates with cholinergic degeneration. Neurology, 81 (18) (2013) 16116.

11. J.M.A. Haworth, Gait, aging and dementia. Reviews in Clinical Gerontology, 18 (01) (2008) 39.

12. L.T. Grinberg, U. Rueb, and H. Heinsen, Brainstem: Neglected locus in neurodegenerative diseases. Front Neurol, 2 (2011) 42.

13. R. Parihar, J.R. Mahoney, and J. Verghese, Relationship of gait and cognition in the elderly. Curr Transl Geriatr Exp Gerontol Rep, 2 (3) (2013).

14. F.A. Sorond, Y. Cruz-Almeida, D.J. Clark, A. Viswanathan, C.R. Scherzer, P. De Jager, et al., Aging, the central nervous system, and mobility in older adults: Neural mechanisms of mobility impairment. J Gerontol A Biol Sci Med Sci, 70 (12) (2015) 1526-32.

15. G.A. Rosenberg, Inflammation and white matter damage in vascular cognitive impairment. Stroke, 40 (3 Suppl) (2009) S20-3.

16. M. Montero-Odasso and V. Hachinski, Preludes to brain failure: Executive dysfunction and gait disturbances. Neurol Sci, 35 (4) (2014) 601-4.

17. A. Viswanathan, W.A. Rocca, and C. Tzourio, Vascular risk factors and dementia: How to move forward? Neurology, 72 (4) (2009) 368-74.

18. M.L. Callisaya, R. Beare, T.G. Phan, L. Blizzard, A.G. Thrift, J. Chen, et al., Brain structural change and gait decline: A longitudinal population-based study. J Am Geriatr Soc, 61 (7) (2013) 1074-9.

19. C. Annweiler and M. Montero-Odasso, Vascular burden as a substrate for higher-level gait disorders in older adults. A review of brain mapping literature. Panminerva Med, 54 (3) (2012) 189-204.

20. M. Montero-Odasso, C. Annweiler, V. Hachinski, A. Islam, N. Yang, and A. Vasudev, Vascular burden predicts gait, mood, and executive function disturbances in older adults with mild cognitive impairment: Results from the gait and brain study. J Am Geriatr Soc, 60 (10) (2012) 1988-90.

21. N. Demnitz, P. Esser, H. Dawes, V. Valkanova, H. Johansen-Berg, K.P. Ebmeier, et al., A systematic review and meta-analysis of cross-sectional studies examining the relationship between mobility and cognition in healthy older adults. Gait Posture, 50 (2016) 164-174.

22. J.M. Hausdorff, G. Yogev, S. Springer, E.S. Simon, and N. Giladi, Walking is more like catching than tapping: Gait in the elderly as a complex cognitive task. Exp Brain Res, 164 (4) (2005) 541-8.

23. R. Holtzer, J. Verghese, X. Xue, and R.B. Lipton, Cognitive processes related to gait velocity: Results from the einstein aging study. Neuropsychology, 20 (2) (2006) 215-23.

24. R. Holtzer, C. Wang, R. Lipton, and J. Verghese, The protective effects of executive functions and episodic memory on gait speed decline in aging defined in the context of cognitive reserve. J Am Geriatr Soc, 60 (11) (2012) 2093-8.

25. R. Holtzer, C. Wang, and J. Verghese, The relationship between attention and gait in aging: Facts and fallacies. Motor Control, 16 (1) (2012) 64-80.

26. K.L. Martin, L. Blizzard, A.G. Wood, V. Srikanth, R. Thomson, L.M. Sanders, et al., Cognitive function, gait, and gait variability in older people: A population-based study. J Gerontol A Biol Sci Med Sci, 68 (6) (2013) 726-32.

27. V.J. Verlinden, J.N. van der Geest, A. Hofman, and M.A. Ikram, Cognition and gait show a distinct pattern of association in the general population. Alzheimers Dement, 10 (3) (2014) 328-35. 
28. O.A. Donoghue, N.F. Horgan, G.M. Savva, H. Cronin, C. O'Regan, and R.A. Kenny, Association between timed up-and-go and memory, executive function, and processing speed. J Am Geriatr Soc, 60 (9) (2012) 1681-6.

29. A. Ble, S. Volpato, G. Zuliani, J.M. Guralnik, S. Bandinelli, F. Lauretani, et al., Executive function correlates with walking speed in older persons: The inchianti study. J Am Geriatr Soc, 53 (3) (2005) 410-5.

30. J. Kaye, N. Mattek, H. Dodge, T. Buracchio, D. Austin, S. Hagler, et al., One walk a year to 1000 within a year: Continuous in-home unobtrusive gait assessment of older adults. Gait Posture, 35 (2) (2012) 197-202.

31. T. ljmker and C.J. Lamoth, Gait and cognition: The relationship between gait stability and variability with executive function in persons with and without dementia. Gait Posture, 35

(1) (2012) 126-30.

32. O. Beauchet, C. Annweiler, M. Montero-Odasso, B. Fantino, F.R. Herrmann, and G. Allali, Gait control: A specific subdomain of executive function? J Neuroeng Rehabil, 9 (2012) 12.

33. J.M. Hausdorff, Gait variability: Methods, modeling and meaning. J Neuroeng Rehabil, 2 (2005) 19.

34. O. Beauchet, G. Allali, M. Montero-Odasso, E. Sejdic, B. Fantino, and C. Annweiler, Motor phenotype of decline in cognitive performance among community-dwellers without dementia: Population-based study and meta-analysis. PLoS One, 9 (6) (2014) e99318.

35. M. Montero-Odasso, S.W. Muir, M. Hall, T.J. Doherty, M. Kloseck, O. Beauchet, et al., Gait variability is associated with frailty in community-dwelling older adults. J Gerontol A Biol Sci Med Sci, 66 (5) (2011) 568-76.

36. K. Duff, J.W. Mold, and M.M. Roberts, Walking speed and global cognition: Results from the oklahoma study. Neuropsychol Dev Cogn B Aging Neuropsychol Cogn, 15 (1) (2008) 31-9.

37. M. Hashimoto, Y. Takashima, A. Uchino, T. Yuzuriha, and H. Yao, Dual task walking reveals cognitive dysfunction in community-dwelling elderly subjects: The sefuri brain mri study. J Stroke Cerebrovasc Dis, 23 (7) (2014) 1770-5.

38. N.M. van Schoor, J.H. Smit, S.M. Pluijm, C. Jonker, and P. Lips, Different cognitive functions in relation to falls among older persons. Immediate memory as an independent risk factor for falls. J Clin Epidemiol, 55 (9) (2002) 855-62.

39. M.B. van lersel, R.P. Kessels, B.R. Bloem, A.L. Verbeek, and M.G. Olde Rikkert, Executive functions are associated with gait and balance in community-living elderly people. J Gerontol A Biol Sci Med Sci, 63 (12) (2008) 1344-9.

40. M.L. Callisaya, L. Blizzard, J.L. McGinley, M.D. Schmidt, and V.K. Srikanth, Sensorimotor factors affecting gait variability in older people--a population-based study. J Gerontol A Biol Sci Med Sci, 65 (4) (2010) 386-92.

41. R.C. Petersen, G.E. Smith, S.C. Waring, R.J. Ivnik, E.G. Tangalos, and E. Kokmen, Mild cognitive impairment: Clinical characterization and outcome. Arch Neurol, 56 (3) (1999) 303-8.

42. E.L. McGough, V.E. Kelly, R.G. Logsdon, S.M. McCurry, B.B. Cochrane, J.M. Engel, et al., Associations between physical performance and executive function in older adults with mild cognitive impairment: Gait speed and the timed "up \& go" test. Phys Ther, 91 (8) (2011) 1198-207.

43. C.C. Persad, J.L. Jones, J.A. Ashton-Miller, N.B. Alexander, and B. Giordani, Executive function and gait in older adults with cognitive impairment. J Gerontol A Biol Sci Med Sci, 63 (12) (2008) 1350-5.

44. P.L. Sheridan, J. Solomont, N. Kowall, and J.M. Hausdorff, Influence of executive function on locomotor function: Divided attention increases gait variability in alzheimer's disease. J Am Geriatr Soc, 51 (11) (2003) 1633-7. 
45. G. Allali, F. Assal, R.W. Kressig, V. Dubost, F.R. Herrmann, and O. Beauchet, Impact of impaired executive function on gait stability. Dement Geriatr Cogn Disord, 26 (4) (2008) 364-9.

46. M.L. Callisaya, C.L. Blizzard, A.G. Wood, A.G. Thrift, T. Wardill, and V.K. Srikanth, Longitudinal relationships between cognitive decline and gait slowing: The tasmanian study of cognition and gait. J Gerontol A Biol Sci Med Sci, 70 (10) (2015) 1226-32.

47. M. Montero-Odasso, A. Oteng-Amoako, M. Speechley, K. Gopaul, O. Beauchet, C. Annweiler, et al., The motor signature of mild cognitive impairment: Results from the gait and brain study. J Gerontol A Biol Sci Med Sci, 69 (11) (2014) 1415-21.

48. J. Verghese, M. Robbins, R. Holtzer, M. Zimmerman, C. Wang, X. Xue, et al., Gait dysfunction in mild cognitive impairment syndromes. J Am Geriatr Soc, 56 (7) (2008) 1244-51.

49. N.T. Aggarwal, R.S. Wilson, T.L. Beck, J.L. Bienias, and D.A. Bennett, Motor dysfunction in mild cognitive impairment and the risk of incident alzheimer disease. Arch Neurol, 63 (12) (2006) 1763-9.

50. T. Doi, H. Shimada, H. Park, H. Makizako, K. Tsutsumimoto, K. Uemura, et al., Cognitive function and falling among older adults with mild cognitive impairment and slow gait. Geriatr Gerontol Int, 15 (8) (2015) 1073-8.

51. J.-S. Choi, H.-S. Oh, D.-W. Kang, K.-R. Mun, M.-H. Choi, S.-J. Lee, et al., Comparison of gait and cognitive function among the elderly with alzheimer's disease, mild cognitive impairment and healthy. International Journal of Precision Engineering and Manufacturing, 12 (1) (2011) 169-173.

52. D. Maquet, F. Lekeu, E. Warzee, S. Gillain, V. Wojtasik, E. Salmon, et al., Gait analysis in elderly adult patients with mild cognitive impairment and patients with mild alzheimer's disease: Simple versus dual task: A preliminary report. Clin Physiol Funct Imaging, 30 (1) (2010) 51-6.

53. I. Tarnanas, S. Papagiannopoulos, D. Kazis, M. Wiederhold, B. Widerhold, and M. Tsolaki, Reliability of a novel serious game using dual-task gait profiles to early characterize amci. Front Aging Neurosci, 7 (2015) 50.

54. S. Boripuntakul, S.R. Lord, M.A. Brodie, S.T. Smith, P. Methapatara, N. Wongpakaran, et al., Spatial variability during gait initiation while dual tasking is increased in individuals with mild cognitive impairment. J Nutr Health Aging, 18 (3) (2014) 307-12.

55. O. Beauchet, G. Allali, C. Launay, F.R. Herrmann, and C. Annweiler, Gait variability at fastpace walking speed: A biomarker of mild cognitive impairment? J Nutr Health Aging, 17 (3) (2013) 235-9.

56. A. Nascimbeni, S. Caruso, A. Salatino, M. Carenza, M. Rigano, A. Raviolo, et al., Dual taskrelated gait changes in patients with mild cognitive impairment. Funct Neurol, 30 (1) (2015) 59-65.

57. A.F. Pettersson, E. Olsson, and L.O. Wahlund, Motor function in subjects with mild cognitive impairment and early alzheimer's disease. Dement Geriatr Cogn Disord, 19 (5-6) (2005) 299304.

58. S.W. Muir, M. Speechley, J. Wells, M. Borrie, K. Gopaul, and M. Montero-Odasso, Gait assessment in mild cognitive impairment and alzheimer's disease: The effect of dual-task challenges across the cognitive spectrum. Gait Posture, 35 (1) (2012) 96-100.

59. L. Bahureksa, B. Najafi, A. Saleh, M. Sabbagh, D. Coon, M.J. Mohler, et al., The impact of mild cognitive impairment on gait and balance: A systematic review and meta-analysis of studies using instrumented assessment. Gerontology, (2016).

60. J. Verghese, C. Wang, R.B. Lipton, R. Holtzer, and X. Xue, Quantitative gait dysfunction and risk of cognitive decline and dementia. J Neurol Neurosurg Psychiatry, 78 (9) (2007) 929-35.

61. M.B. van lersel, W. Hoefsloot, M. Munneke, B.R. Bloem, and M.G. Olde Rikkert, Systematic review of quantitative clinical gait analysis in patients with dementia. Z Gerontol Geriatr, 37 (1) (2004) 27-32. 
62. L.M. Allan, C.G. Ballard, D.J. Burn, and R.A. Kenny, Prevalence and severity of gait disorders in alzheimer's and non-alzheimer's dementias. J Am Geriatr Soc, 53 (10) (2005) 1681-7.

63. E. Scherder, L. Eggermont, J. Sergeant, and F. Boersma, Physical activity and cognition in alzheimer's disease: Relationship to vascular risk factors, executive functions and gait. Rev Neurosci, 18 (2) (2007) 149-58.

64. E.L. McGough, B.B. Cochrane, K.C. Pike, R.G. Logsdon, S.M. McCurry, and L. Teri, Dimensions of physical frailty and cognitive function in older adults with amnestic mild cognitive impairment. Ann Phys Rehabil Med, 56 (5) (2013) 329-41.

65. G. McKhann, D. Drachman, M. Folstein, R. Katzman, D. Price, and E.M. Stadlan, Clinical diagnosis of alzheimer's disease: Report of the nincds-adrda work group under the auspices of department of health and human services task force on alzheimer's disease. Neurology, 34 (7) (1984) 939-44.

66. T.A. Ala and W.H. Frey, 2 nd, Validation of the nincds-adrda criteria regarding gait in the clinical diagnosis of alzheimer disease. A clinicopathologic study. Alzheimer Dis Assoc Disord, 9 (3) (1995) 152-9.

67. V. Mazoteras Munoz, G. Abellan van Kan, C. Cantet, F. Cortes, P.J. Ousset, Y. Rolland, et al., Gait and balance impairments in alzheimer disease patients. Alzheimer Dis Assoc Disord, 24 (1) (2010) 79-84.

68. A.F. Pettersson, M. Engardt, and L.O. Wahlund, Activity level and balance in subjects with mild alzheimer's disease. Dement Geriatr Cogn Disord, 13 (4) (2002) 213-6.

69. A. Castrillo, L.M. Olmos, F. Rodriguez, and J. Duarte, Gait disorder in a cohort of patients with mild and moderate alzheimer's disease. Am J Alzheimers Dis Other Demen, 31 (3) (2016) 257-62.

70. O. Beauchet, G. Allali, G. Berrut, C. Hommet, V. Dubost, and F. Assal, Gait analysis in demented subjects: Interests and perspectives. Neuropsychiatr Dis Treat, 4 (1) (2008) 15560.

71. R. Camicioli and L. Licis, Motor impairment predicts falls in specialized alzheimer care units. Alzheimer Dis Assoc Disord, 18 (4) (2004) 214-8.

72. E. Scherder, L. Eggermont, D. Swaab, M. van Heuvelen, Y. Kamsma, M. de Greef, et al., Gait in ageing and associated dementias; its relationship with cognition. Neurosci Biobehav Rev, 31 (4) (2007) 485-97.

73. D. Morgan, M. Funk, M. Crossley, J. Basran, A. Kirk, and V. Dal Bello-Haas, The potential of gait analysis to contribute to differential diagnosis of early stage dementia: Current research and future directions. Can J Aging, 26 (1) (2007) 19-32.

74. T. Nakamura, [variability in gait among patients with alzheimer-type dementia]. Nihon Ronen Igakkai Zasshi, 33 (11) (1996) 816-9.

75. K.E. Webster, J.R. Merory, and J.E. Wittwer, Gait variability in community dwelling adults with alzheimer disease. Alzheimer Dis Assoc Disord, 20 (1) (2006) 37-40.

76. C.J. Lamoth, F.J. van Deudekom, J.P. van Campen, B.A. Appels, O.J. de Vries, and M. Pijnappels, Gait stability and variability measures show effects of impaired cognition and dual tasking in frail people. J Neuroeng Rehabil, 8 (2011) 2.

77. S. Gillain, E. Warzee, F. Lekeu, V. Wojtasik, D. Maquet, J.L. Croisier, et al., The value of instrumental gait analysis in elderly healthy, mci or alzheimer's disease subjects and a comparison with other clinical tests used in single and dual-task conditions. Ann Phys Rehabil Med, 52 (6) (2009) 453-74.

78. G. Cocchini, S. Della Sala, R.H. Logie, R. Pagani, L. Sacco, and H. Spinnler, Dual task effects of walking when talking in alzheimer's disease. Rev Neurol (Paris), 160 (1) (2004) 74-80.

79. H. Visser, Gait and balance in senile dementia of alzheimer's type. Age Ageing, 12 (4) (1983) 296-301. 
80. G. Allali, R.W. Kressig, F. Assal, F.R. Herrmann, V. Dubost, and O. Beauchet, Changes in gait while backward counting in demented older adults with frontal lobe dysfunction. Gait Posture, 26 (4) (2007) 572-6.

81. M.B. van lersel, A.L. Verbeek, B.R. Bloem, M. Munneke, R.A. Esselink, and M.G. Rikkert, Frail elderly patients with dementia go too fast. J Neurol Neurosurg Psychiatry, 77 (7) (2006) 8746.

82. N. Saji, N. Ogama, K. Toba, and T. Sakurai, White matter hyperintensities and geriatric syndrome: An important role of arterial stiffness. Geriatr Gerontol Int, 15 Suppl 1 (2015) 1725.

83. J.J. Zheng, K. Delbaere, J.C. Close, P.S. Sachdev, and S.R. Lord, Impact of white matter lesions on physical functioning and fall risk in older people: A systematic review. Stroke, 42 (7) (2011) 2086-90.

84. J.J. Zheng, S.R. Lord, J.C. Close, P.S. Sachdev, W. Wen, H. Brodaty, et al., Brain white matter hyperintensities, executive dysfunction, instability, and falls in older people: A prospective cohort study. J Gerontol A Biol Sci Med Sci, 67 (10) (2012) 1085-91.

85. V. Srikanth, R. Beare, L. Blizzard, T. Phan, J. Stapleton, J. Chen, et al., Cerebral white matter lesions, gait, and the risk of incident falls: A prospective population-based study. Stroke, 40 (1) (2009) 175-80.

86. M.L. Callisaya, R. Beare, T. Phan, L. Blizzard, A.G. Thrift, J. Chen, et al., Progression of white matter hyperintensities of presumed vascular origin increases the risk of falls in older people. J Gerontol A Biol Sci Med Sci, 70 (3) (2015) 360-6.

87. K.F. de Laat, A.G. van Norden, R.A. Gons, L.J. van Oudheusden, I.W. van Uden, B.R. Bloem, et al., Gait in elderly with cerebral small vessel disease. Stroke, 41 (8) (2010) 1652-8.

88. M.N. Lopez, R.A. Charter, B. Mostafavi, L.P. Nibut, and W.E. Smith, Psychometric properties of the folstein mini-mental state examination. Assessment, 12 (2) (2005) 137-44.

89. H. Baezner, C. Blahak, A. Poggesi, L. Pantoni, D. Inzitari, H. Chabriat, et al., Association of gait and balance disorders with age-related white matter changes: The ladis study. Neurology, 70 (12) (2008) 935-42.

90. G.C. Roman, T.K. Tatemichi, T. Erkinjuntti, J.L. Cummings, J.C. Masdeu, J.H. Garcia, et al., Vascular dementia: Diagnostic criteria for research studies. Report of the ninds-airen international workshop. Neurology, 43 (2) (1993) 250-60.

91. H. Bazner, M. Oster, M. Daffertshofer, and M. Hennerici, Assessment of gait in subcortical vascular encephalopathy by computerized analysis: A cross-sectional and longitudinal study. J Neurol, 247 (11) (2000) 841-9.

92. R. Moretti, M. Cavressi, and P. Tomietto, Gait and apathy as relevant symptoms of subcortical vascular dementia. Am J Alzheimers Dis Other Demen, 30 (4) (2015) 390-9.

93. M.I. Tolea, J.C. Morris, and J.E. Galvin, Trajectory of mobility decline by type of dementia. Alzheimer Dis Assoc Disord, 30 (1) (2016) 60-6.

94. M. Montero-Odasso, J. Verghese, O. Beauchet, and J.M. Hausdorff, Gait and cognition: A complementary approach to understanding brain function and the risk of falling. J Am Geriatr Soc, 60 (11) (2012) 2127-36.

95. T.Y. Liu-Ambrose, M.C. Ashe, P. Graf, B.L. Beattie, and K.M. Khan, Increased risk of falling in older community-dwelling women with mild cognitive impairment. Phys Ther, 88 (12) (2008) 1482-91.

96. K. Delbaere, N.A. Kochan, J.C. Close, J.C. Menant, D.L. Sturnieks, H. Brodaty, et al., Mild cognitive impairment as a predictor of falls in community-dwelling older people. Am J Geriatr Psychiatry, 20 (10) (2012) 845-53.

97. S. Tyrovolas, A. Koyanagi, E. Lara, Z.I. Santini, and J.M. Haro, Mild cognitive impairment is associated with falls among older adults: Findings from the irish longitudinal study on ageing (tilda). Exp Gerontol, 75 (2016) 42-7. 
98. L.M. Allan, C.G. Ballard, E.N. Rowan, and R.A. Kenny, Incidence and prediction of falls in dementia: A prospective study in older people. PLoS One, 4 (5) (2009) e5521.

99. O. Beauchet, C. Annweiler, V. Dubost, G. Allali, R.W. Kressig, S. Bridenbaugh, et al., Stops walking when talking: A predictor of falls in older adults? Eur J Neurol, 16 (7) (2009) 786-95.

100. O. Beauchet, C. Annweiler, G. Allali, G. Berrut, F.R. Herrmann, and V. Dubost, Recurrent falls and dual task-related decrease in walking speed: Is there a relationship? J Am Geriatr Soc, 56 (7) (2008) 1265-9.

101. J. Muhaidat, A. Kerr, J.J. Evans, M. Pilling, and D.A. Skelton, Validity of simple gait-related dual-task tests in predicting falls in community-dwelling older adults. Arch Phys Med Rehabil, 95 (1) (2014) 58-64.

102. M.E. Taylor, M.M. Ketels, K. Delbaere, S.R. Lord, A.S. Mikolaizak, and J.C. Close, Gait impairment and falls in cognitively impaired older adults: An explanatory model of sensorimotor and neuropsychological mediators. Age Ageing, 41 (5) (2012) 665-9.

103. M. Yamada, T. Aoyama, H. Arai, K. Nagai, B. Tanaka, K. Uemura, et al., Dual-task walk is a reliable predictor of falls in robust elderly adults. J Am Geriatr Soc, 59 (1) (2011) 163-4.

104. H.G. Kang, L. Quach, W. Li, and L.A. Lipsitz, Stiffness control of balance during dual task and prospective falls in older adults: The mobilize boston study. Gait Posture, 38 (4) (2013) 75763.

105. S.W. Muir, K. Gopaul, and M.M. Montero Odasso, The role of cognitive impairment in fall risk among older adults: A systematic review and meta-analysis. Age Ageing, 41 (3) (2012) 299-308.

106. T.Y. Chen, C.L. Peronto, and J.D. Edwards, Cognitive function as a prospective predictor of falls. J Gerontol B Psychol Sci Soc Sci, 67 (6) (2012) 720-8.

107. M.E. Taylor, K. Delbaere, S.R. Lord, A.S. Mikolaizak, H. Brodaty, and J.C. Close, Neuropsychological, physical, and functional mobility measures associated with falls in cognitively impaired older adults. J Gerontol A Biol Sci Med Sci, 69 (8) (2014) 987-95.

108. C.S. Sterke, E.F. van Beeck, C.W. Looman, R.W. Kressig, and T.J. van der Cammen, An electronic walkway can predict short-term fall risk in nursing home residents with dementia. Gait Posture, 36 (1) (2012) 95-101.

109. J. Verghese, C. Derby, M.J. Katz, and R.B. Lipton, High risk neurological gait syndrome and vascular dementia. J Neural Transm (Vienna), 114 (10) (2007) 1249-52.

110. J. Verghese, R.B. Lipton, C.B. Hall, G. Kuslansky, M.J. Katz, and H. Buschke, Abnormality of gait as a predictor of non-alzheimer's dementia. N Engl J Med, 347 (22) (2002) 1761-8.

111. J. Dumurgier, F. Artaud, C. Touraine, O. Rouaud, B. Tavernier, C. Dufouil, et al., Gait speed and decline in gait speed as predictors of incident dementia. J Gerontol A Biol Sci Med Sci, (2016).

112. G. Abellan van Kan, Y. Rolland, S. Gillette-Guyonnet, V. Gardette, C. Annweiler, O. Beauchet, et al., Gait speed, body composition, and dementia. The epidos-toulouse cohort. J Gerontol A Biol Sci Med Sci, 67 (4) (2012) 425-32.

113. S. Marquis, M.M. Moore, D.B. Howieson, G. Sexton, H. Payami, J.A. Kaye, et al., Independent predictors of cognitive decline in healthy elderly persons. Arch Neurol, 59 (4) (2002) 601-6.

114. G. Abellan van Kan, Y. Rolland, S. Andrieu, J. Bauer, O. Beauchet, M. Bonnefoy, et al., Gait speed at usual pace as a predictor of adverse outcomes in community-dwelling older people an international academy on nutrition and aging (iana) task force. J Nutr Health Aging, 13 (10) (2009) 881-9.

115. J. Dumurgier, A. Elbaz, P. Ducimetiere, B. Tavernier, A. Alperovitch, and C. Tzourio, Slow walking speed and cardiovascular death in well functioning older adults: Prospective cohort study. Bmj, 339 (2009) b4460.

116. S. Studenski, S. Perera, K. Patel, C. Rosano, K. Faulkner, M. Inzitari, et al., Gait speed and survival in older adults. Jama, 305 (1) (2011) 50-8. 
117. O. Beauchet, C. Annweiler, M.L. Callisaya, A.M. De Cock, J.L. Helbostad, R.W. Kressig, et al., Poor gait performance and prediction of dementia: Results from a meta-analysis. J Am Med Dir Assoc, 17 (6) (2016) 482-90.

118. R. Morris, S. Lord, J. Bunce, D. Burn, and L. Rochester, Gait and cognition: Mapping the global and discrete relationships in ageing and neurodegenerative disease. Neurosci Biobehav Rev, 64 (2016) 326-45.

119. L.H. Kikkert, N. Vuillerme, J.P. van Campen, T. Hortobagyi, and C.J. Lamoth, Walking ability to predict future cognitive decline in old adults: A scoping review. Ageing Res Rev, 27 (2016) 114.

120. T. Buracchio, H.H. Dodge, D. Howieson, D. Wasserman, and J. Kaye, The trajectory of gait speed preceding mild cognitive impairment. Arch Neurol, 67 (8) (2010) 980-6.

121. J. Verghese, C. Wang, R.B. Lipton, and R. Holtzer, Motoric cognitive risk syndrome and the risk of dementia. J Gerontol A Biol Sci Med Sci, 68 (4) (2013) 412-8.

122. J. Verghese, C. Annweiler, E. Ayers, N. Barzilai, O. Beauchet, D.A. Bennett, et al., Motoric cognitive risk syndrome: Multicountry prevalence and dementia risk. Neurology, 83 (8) (2014) 718-26.

123. M. Hamer and Y. Chida, Physical activity and risk of neurodegenerative disease: A systematic review of prospective evidence. Psychol Med, 39 (1) (2009) 3-11.

124. J.G. van Uffelen, A.P.M.J. Chin, M. Hopman-Rock, and W. van Mechelen, The effects of exercise on cognition in older adults with and without cognitive decline: A systematic review. Clin J Sport Med, 18 (6) (2008) 486-500.

125. H. Ohman, N. Savikko, T.E. Strandberg, and K.H. Pitkala, Effect of physical exercise on cognitive performance in older adults with mild cognitive impairment or dementia: $A$ systematic review. Dement Geriatr Cogn Disord, 38 (5-6) (2014) 347-65.

126. G. Zheng, R. Xia, W. Zhou, J. Tao, and L. Chen, Aerobic exercise ameliorates cognitive function in older adults with mild cognitive impairment: A systematic review and metaanalysis of randomised controlled trials. Br J Sports Med, (2016).

127. V. Valkanova, R. Eguia Rodriguez, and K.P. Ebmeier, Mind over matter--what do we know about neuroplasticity in adults? Int Psychogeriatr, 26 (6) (2014) 891-909.

128. M. Montero-Odasso, J.L. Wells, M.J. Borrie, and M. Speechley, Can cognitive enhancers reduce the risk of falls in older people with mild cognitive impairment? A protocol for a randomised controlled double blind trial. BMC Neurol, 9 (2009) 42.

129. M. Montero-Odasso, S.W. Muir-Hunter, A. Oteng-Amoako, K. Gopaul, A. Islam, M. Borrie, et al., Donepezil improves gait performance in older adults with mild alzheimer's disease: $A$ phase ii clinical trial. J Alzheimers Dis, 43 (1) (2015) 193-9.

130. F. Assal, G. Allali, R.W. Kressig, F.R. Herrmann, and O. Beauchet, Galantamine improves gait performance in patients with alzheimer's disease. J Am Geriatr Soc, 56 (5) (2008) 946-7.

131. O. Beauchet, C.P. Launay, G. Allali, G. Watfa, K. Gallouj, F.R. Herrmann, et al., Anti-dementia drugs and changes in gait: A pre-post quasi-experimental pilot study. BMC Neurol, 13 (2013) 184.

132. O. Beauchet, G. Allali, C. Launay, B. Fantino, and C. Annweiler, Does memantine improve the gait of individuals with alzheimer's disease? J Am Geriatr Soc, 59 (11) (2011) 2181-2.

133. O. Beauchet, C.P. Launay, G. Allali, and C. Annweiler, Changes in gait variability with antidementia drugs: A systematic review and meta-analysis. CNS Drugs, 28 (6) (2014) 513-8.

134. X. Liao, G. Li, A. Wang, T. Liu, S. Feng, Z. Guo, et al., Repetitive transcranial magnetic stimulation as an alternative therapy for cognitive impairment in alzheimer's disease: $\mathrm{A}$ meta-analysis. J Alzheimers Dis, 48 (2) (2015) 463-72.

135. A. Wagle Shukla, J.J. Shuster, J.W. Chung, D.E. Vaillancourt, C. Patten, J. Ostrem, et al., Repetitive transcranial magnetic stimulation ( $\mathrm{rtms}$ ) therapy in parkinson disease: A metaanalysis. Pm r, 8 (4) (2016) 356-66. 
136. W. Klomjai, A. Lackmy-Vallee, N. Roche, P. Pradat-Diehl, V. Marchand-Pauvert, and R. Katz, Repetitive transcranial magnetic stimulation and transcranial direct current stimulation in motor rehabilitation after stroke: An update. Ann Phys Rehabil Med, 58 (4) (2015) 220-4. 